\title{
Longitudinal follow-up of quadriceps strength and function in a COPD cohort after 3 years
}

\author{
To the Editor:
}

Skeletal muscle weakness is a distinct extrapulmonary manifestation of chronic obstructive pulmonary disease (COPD), which is associated with a poor prognosis [1] and may represent a novel therapeutic target [2]. However, there are only sparse longitudinal data from prospective studies on skeletal muscle weakness and its relationship with disease progression in COPD. Previous studies have been cross-sectional in nature, and have failed to correlate data with baseline measures of exercise performance or systemic inflammation.

The ERICA (Evaluation of the Role of Inflammation in Chronic Airways disease) trial was a multicentre prospective study in which quadriceps maximal voluntary contraction force (QMVC) was assessed in addition to other measures to provide a detailed evaluation of the extrapulmonary manifestations of COPD [3]. Following ethics approval (NRES Harrow: 11/LO/1636) at a single site, a subset of these patients underwent more detailed evaluation with the aim of relating fibre type to phenotypic parameters (ClinicalTrials.gov number NCT01471587); the primary result has been presented elsewhere [4], and here we present follow-up data for the clinical parameters over a 3 -year period.

Briefly, 61 subjects were studied at baseline; key inclusion criteria were a clinical diagnosis of COPD confirmed by post-bronchodilator spirometry (forced expiratory volume in 1 s/forced vital capacity (FEV 1 / FVC) ratio $<70 \%$ and $\mathrm{FEV}_{1}<80 \%$ predicted) and a $>10$ pack-year smoking history. Patients with development of exacerbation within 4 weeks of screening, or a history of other respiratory diagnoses or exercise-limiting musculoskeletal or neurological disease, were excluded. After 3 years, participants were invited for follow-up, at which the baseline assessments were repeated. Key assessments included demographics, anthropometrics, body composition (measured as bioimpedance), post-bronchodilator spirometry and maximal sniff nasal inspiratory pressure (SNIP), physical activity (Sensewear Pro 3 armband (BodyMedia Inc, Pittsburgh, PA, USA)), Short Physical Performance Battery (SPPB), 6 minute walk test (6MWT), aortic pulse wave velocity (aPWV), QMVC, ultrasound-determined rectus femoris muscle cross-sectional area $\left(\mathrm{RF}_{\mathrm{CSA}}\right)$, systemic inflammatory markers (fibrinogen, white cell count (WCC) and high-sensitivity C-reactive protein (hsCRP)) and health-related quality of life questionnaires. Mean change between baseline and follow-up, and rate of change per year were calculated and analysed using paired $t$-test. Graphpad Prism (version 5; GraphPad, La Jolla, CA, USA) was used for statistical analysis, and significance was accepted as $\mathrm{p}<0.05$.

In total, 31 participants (61\% male) returned for follow-up; values are expressed as mean (SD) (table 1). Reasons for not returning were: declined follow-up for personal reasons (12, 20\%), death, (10, 16\%), inability to attend the study centre due to deterioration in $\operatorname{COPD}(6,10 \%)$, lung transplant surgery $(1,1 \%)$ and being uncontactable $(1,1 \%)$ (figure 1$)$. Nonreturning participants were more nutritionally depleted judged by both body mass index (BMI) $(p=0.002)$ and fat-free mass index (FFMI) $(p=0.03)$, and tended to poorer lung function at baseline judged by $\mathrm{FEV}_{1} \%$ predicted $(\mathrm{p}=0.08)$.

At baseline, the restudied subjects were aged 67 (8) years, with a 52 (40) pack-year smoking history, FEV1 47.0 (20.2) \% predicted, and Medical Research Council dyspnoea score of 2.8 (1.3). Four participants were current smokers at baseline; of these, two were still smoking at the time of follow-up assessment.

@ERSpublications

The short physical performance battery may be a useful endpoint in assessing impact on skeletal muscle dysfunction http://ow.ly/xf8z30dOFMz

Cite this article as: Buttery SC, Mohan D, Fisk M, et al. Longitudinal follow-up of quadriceps strength and function in a COPD cohort after 3 years. Eur Respir J 2017; 50: 1700707 [https://doi.org/10.1183/ 13993003.00707-2017]. 
TABLE 1 Clinical characteristics at baseline and follow-up

\begin{tabular}{|c|c|c|c|c|c|c|c|}
\hline & \multicolumn{2}{|c|}{ Baseline } & \multirow{2}{*}{$\begin{array}{l}\text { Follow-up } \\
\text { at } 3 \text { years }(n=31)\end{array}$} & \multicolumn{2}{|l|}{ Change } & \multirow[t]{2}{*}{$95 \% \mathrm{Cl}^{\#}$} & \multirow[t]{2}{*}{ p-value } \\
\hline & No follow-up (n=30) & Returning ( $n=31$ ) & & Baseline to follow-up & Per year & & \\
\hline \multicolumn{8}{|l|}{ Demographics } \\
\hline Age years & $65(9)$ & $67(8)$ & $70(8)$ & - & - & - & - \\
\hline Male $\%$ & 77.3 & 61.3 & 61.3 & - & - & - & - \\
\hline Current smoker \% & 10.0 & 12.9 & 6.5 & - & - & - & - \\
\hline Smoking history pack years & $40(20)$ & 52 (40) & $52(40)$ & - & - & - & - \\
\hline $\mathrm{BMI} \mathrm{kg} \cdot \mathrm{m}^{-2}$ & $22.6(4.7)^{9}$ & $26.7(5.1)$ & $26.7(6.0)$ & $-0.01(1.66)$ & 0.00 & -0.62 to 0.60 & 0.1 \\
\hline MRC dyspnoea score (out of 5) & $3(1.4)$ & $3(1.1)$ & $3(1.3)$ & $-0.16(1.24)$ & -0.05 & -0.55 to 0.28 & 0.5 \\
\hline \multicolumn{8}{|l|}{ Respiratory measurements } \\
\hline FEV $1 \%$ predicted & $38.3(17.5)$ & $47.0(20.2)$ & $42.8(21.5)$ & $-5.56(15.0)$ & -1.62 & -5.81 to -0.54 & 0.02 \\
\hline $\mathrm{FEV}_{1} / \mathrm{FVC}$ & $0.34(0.14)$ & $0.38(0.16)$ & $0.40(0.16)$ & $0.01(0.16)$ & 0.00 & -0.01 to 0.07 & 0.1 \\
\hline Exacerbations per year & $2.1(2.4)$ & $1.8(2.0)$ & $2.1(2.2)$ & $0.26(2.85)$ & 0.07 & -0.82 to 1.35 & 0.6 \\
\hline \multicolumn{8}{|l|}{ Muscle indices } \\
\hline 6MWD min & $408(116)$ & 399 (132) & 325 (172) & $-71.7(162)$ & -16.8 & -119 to -27.4 & 0.003 \\
\hline SPPB total score (out of 12 ) & 10 (1.9) & $11(1.4)$ & $9(3.0)$ & $-1.42(2.99)$ & -0.45 & -2.70 to -0.40 & 0.01 \\
\hline Balance score (out of 4) & $4.0(0.4)$ & $4.0(0.2)$ & $3.7(1.0)$ & $-0.39(1.21)$ & -0.1 & -0.84 to 0.06 & 0.09 \\
\hline Gait speed score (out of 4) & $3.8(0.4)$ & $3.8(0.5)$ & $3.4(1.1)$ & $-0.39(1.09)$ & -0.1 & -0.79 to 0.01 & 0.06 \\
\hline $4 \mathrm{~m}$ gait speed time $\mathrm{s}$ & $4.2(0.8)$ & $4.2(0.9)$ & $4.3(1.5)$ & $-0.03(1.59)$ & -0.1 & -0.64 to 0.45 & 0.7 \\
\hline Chair-stand score (out of 4) & $2.6(1.4)^{\pi}$ & $3.1(1.0)$ & $2.3(1.3)$ & $-0.87(1.31)$ & -0.3 & -1.35 to -0.39 & 0.0009 \\
\hline Chair-stand time $s$ & $16.1(13.3)^{\pi}$ & $13.8(9.4)$ & $19.3(14.7)$ & $5.46(15.8)$ & 1.6 & 0.47 to 13.1 & 0.05 \\
\hline FFM kg & $46.8(9.1)^{\pi}$ & $52.1(9.8)$ & $50.3(11.4)$ & $-1.80(3.47)$ & -0.58 & -3.08 to -0.53 & $<0.01$ \\
\hline FFMI & $14.3(3.4)$ & $16.3(2.2)$ & $16.3(3.2)$ & $0.06(1.66)$ & 0.02 & -0.54 to 0.67 & 0.8 \\
\hline QMVC kg & $28.8(9.5)$ & $32.6(8.0)$ & $27.2(9.0)$ & $-5.20(11.2)$ & -1.48 & -7.19 to -2.90 & $<0.0001$ \\
\hline QMVC \% predicted & $68.8(29.7)$ & $72.7(16.7)$ & $64.8(18.8)$ & $-7.39(12.9)$ & -2.20 & -12.3 to -2.50 & $<0.01$ \\
\hline $\mathrm{RF}_{\mathrm{CSA}} \mathrm{mm}^{2}$ & $516(184)$ & $603(136)$ & $449(126)$ & $-154(245)$ & -42.2 & -181 to -99.8 & $<0.0001$ \\
\hline SNIP & $56.0(19.2)$ & $53.7(24.2)$ & $60.6(19.2)$ & $4.6(20.1)$ & 1.94 & -2.760 to 11.99 & 0.2 \\
\hline \multicolumn{8}{|l|}{ Cardiovascular indices } \\
\hline Systolic BP mmHg & 132 (14.2) & $140(16.3)$ & $136(16.3)$ & $-3.97(19.3)$ & -1.39 & -11.1 to 3.12 & 0.7 \\
\hline $\mathrm{aPWV} \mathrm{m} \cdot \mathrm{s}^{-1}$ & $10.6(3.5)$ & $10.7(2.7)$ & $10.3(2.7)$ & $-2.35(5.63)$ & -0.66 & -1.44 to 0.96 & 0.7 \\
\hline \multicolumn{8}{|l|}{ Inflammatory markers } \\
\hline Fibrinogen $\mathrm{g} \cdot \mathrm{dL}^{-1}$ & $3.8(0.9)$ & $3.4(0.7)$ & $3.8(0.8)$ & $0.35(0.51)$ & 0.11 & 0.16 to 0.54 & $<0.001$ \\
\hline$W C C \times 10^{9} \cdot L^{-1}$ & $7.2(1.7)$ & $8.0(2.9)$ & $7.9(2.3)$ & $-0.10(2.62)$ & 0.02 & -10.5 to 4.66 & 0.4 \\
\hline $\mathrm{hsCRP} \mathrm{mg} \cdot \mathrm{L}^{-1}$ & 9.8 (16.3) & $11.4(22.5)$ & $8.1(19.6)$ & $-13.1(22.1)$ & -4.02 & -0.86 to 1.06 & 0.8 \\
\hline
\end{tabular}

Baseline, follow-up and change data are expressed as mean (standard deviation) unless otherwise stated. BMI: body mass index; MRC: Medical Research Council; FEV1: forced expiratory volume in $1 \mathrm{~s}$; FVC: forced vital capacity; 6MWD: 6-min walk distance; SPPB: short physical performance battery; FFM: fat-free mass; FFMI: fat-free mass index; QMVC: quadriceps maximal voluntary contraction; RFCSA: rectus femoris (cross-sectional area); SNIP: maximal sniff nasal inspiratory pressure; BP: blood pressure; aPWV: aortic pulse wave velocity; WCC: white cell count; hsCRP: high-sensitivity C-reactive protein. \# : confidence interval based on change from baseline to follow-up; ${ }^{\text {? }}$ : baseline measure differed significantly between the returning cohort and the non-follow-up cohort. 
FIGURE 1 Pie chart showing the reasons for subjects declining follow-up at 3 years.

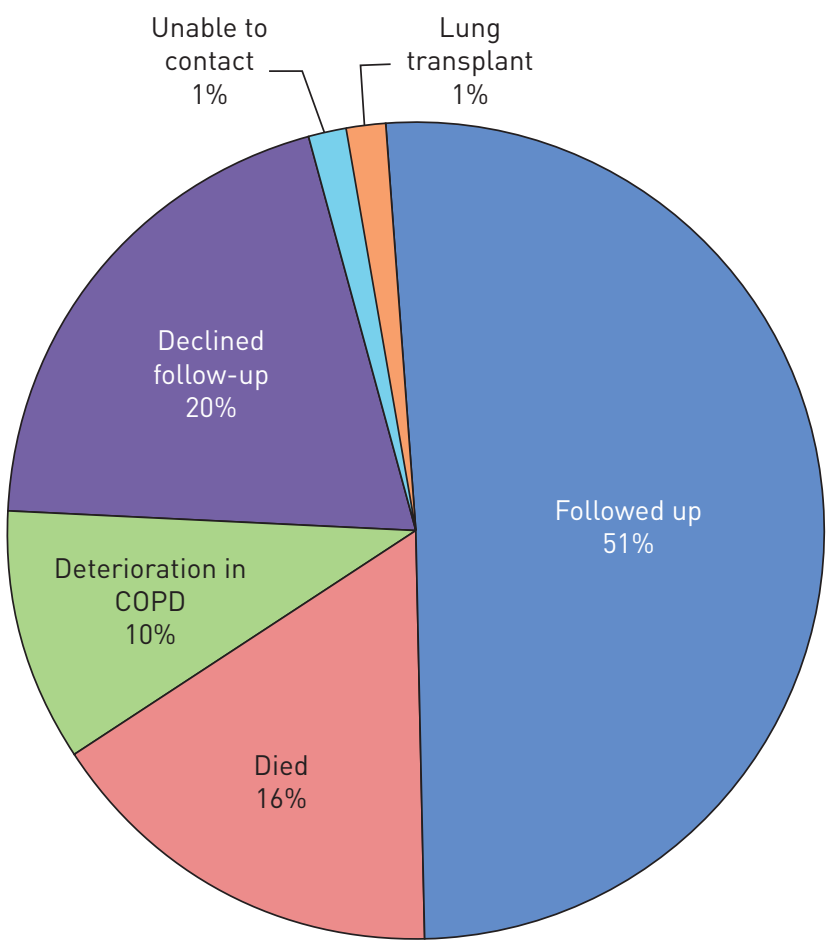

Statistically significant declines were observed in QMVC $(-1.48 \mathrm{~kg}$ per year; $95 \%$ CI -7.19 to -2.90 , $\mathrm{p} \leqslant 0.0001), \mathrm{RF}_{\mathrm{CSA}}\left(-42.2 \mathrm{~mm}^{2}\right.$ per year; $95 \% \mathrm{CI}-181$ to $\left.-99.8, \mathrm{p} \geqslant 0.0001\right)$ and fat-free mass (FFM) $(-0.58 \mathrm{~kg}$ per year; $95 \% \mathrm{CI}-3.08$ to $-0.53, \mathrm{p}=0.007)$ over 3 years, without change in BMI. There was no relationship between sex and QMVC change over time $(\mathrm{r}=0.03, \mathrm{p}=0.34)$, and linear regression showed no correlation between QMVC decline and either comorbidities $(r=0.32, p=0.9)$, corticosteroid treatment $(\mathrm{r}=0.04, \mathrm{p}=0.8)$, frequent exacerbations $(\mathrm{r}=0.045, \mathrm{p}=0.9)$ or hospital admissions $(\mathrm{r}=0.009, \mathrm{p}=0.96)$. The magnitude of change was consistent with our earlier study [5], which found a reduction in quadriceps strength of $1.5 \mathrm{~kg}$ over 1 year in patients with COPD. The changes in FFM may be explained by skeletal muscle loss, which is well documented in COPD and consistent with the observed decline in both QMVC and $\mathrm{RF}_{\mathrm{CSA}}$ changes. Although there was no correlation between change in $\mathrm{RF}_{\mathrm{CSA}}$ and QMVC, there was a correlation between change in $\mathrm{RF}_{\mathrm{CSA}}$ and change in FFM $(\mathrm{r}=0.22 ; \mathrm{p}=0.007)$.

As well as loss of skeletal muscle mass, we also observed a significant decline in performance measures over 3 years. In particular, the change in 6MWD showed a linear relationship with change in QMVC $(\mathrm{r}=0.52 ; \mathrm{p}=0.003)$ but not with change in $\mathrm{RF}_{\mathrm{CSA}}(\mathrm{r}=0.27 ; \mathrm{p}=0.19)$, with a similar order of magnitude to that previously reported in the ECLIPSE (Evaluation of COPD Longitudinally to Identify Predictive Surrogate Endpoint) cohort, and which over 3 years exceeded the minimally clinically important difference (MCID) [5]. Similarly, the SPPB score significantly decreased from baseline to follow-up; of the three individual components, the sit-to-stand test showed the greatest (and the only statistically significant) contribution to this decline; this is consistent with the data of Bernabeu-Mora et al. [6], which suggested that only the five-repetition sit-to-stand test subcomponent of the SPPB was a discriminative measure of self-reported mobility limitation. This is of importance as the European Medical Agency has recently indicated a favourable opinion for the SPPB as an endpoint in clinical trials for sarcopenia, yet little is known about how SPPB scores change over time in COPD. We acknowledge that a weakness of the current study is that we did not study healthy age-matched controls. However, available data suggest that progression in SPPB in our cohort ( 0.45 points/year) is faster than that reported in healthy seniors (0.1-0.3 points/year) [7].

Our study failed to replicate the earlier findings of Bernabeu-Mora et al. [6] and our previous study [8] of a correlation between SPPB scores and quadriceps strength, and we also observed no correlation between change in $\mathrm{RF}_{\mathrm{CSA}}$ and individual components of the SPPB. Several reasons could explain this, including the small sample size and the fact that our cohort was highly functioning at baseline (SPPB $>10)$, giving a limited spread of SPPB values. However, we note that the annualised change observed in the sit-to-stand test exceeded the time (1.7 s) that we have previously established as the MCID for this test in COPD [9]. Thus the SPPB, or its sit-to-stand component, represents a relatively simple clinical outcome measure that 
can identify patients at risk of muscle weakness, can be used as a tool in inpatient and outpatient environments without the need for specialist equipment or personnel, and can detect change in a relatively small cohort.

In summary, our data, despite the small sample size and likely differential subject drop-outs, show that a measurable decline in quadriceps strength and mass is observed over 3 years in patients with COPD, which in the case of QMVC correlates with clinically relevant changes in 6MWT and SPPB. Consistent with this, change in SPPB was principally driven by the chair-stand element, which we have previously shown to be related to quadriceps strength [10]. Given the known predictive power of the SPPB for functional decline, hospitalisation and mortality [8], our data suggest that skeletal muscle dysfunction is an appropriate target for intervention in COPD, and that SPPB can be a useful endpoint in clinical trials assessing impact on skeletal muscle dysfunction.

Sara C. Buttery ${ }^{1,2}$, Divya Mohan ${ }^{1,2,3}$, Marie Fisk ${ }^{4,5}$, Nicholas S. Hopkinson $\oplus^{1,2}$, Ruth Tal-Singer ${ }^{3}$, Ian B. Wilkinson ${ }^{4,5}$ and Michael I. Polkey,

${ }^{1}$ NIHR Respiratory Biomedical Research Unit, Royal Brompton \& Harefield NHS Foundation Trust, London, UK. ${ }^{2}$ NHLI, Respiratory Medicine, Imperial College, London, UK. ${ }^{3}$ Research and Development, GlaxoSmithKline, King of Prussia, PA, USA. ${ }^{4}$ Division of Experimental Medicine and Immunotherapeutics, University of Cambridge, Cambridge, UK. ${ }^{5}$ Cambridge Clinical Trials Unit (CCTU), Addenbrooke's Hospital, Cambridge, UK.

Correspondence: S.C. Buttery, NIHR Respiratory Biomedical Research Unit, Royal Brompton \& Harefield NHS Foundation Trust, Fulham Road, London SW3 6NP, UK. E-mail: s.buttery@rbht.nhs.uk

Received: Jan 312017 | Accepted after revision: May 042017

This study is registered at ClinicalTrials.gov with identifier number NCT01471587.

Support statement: This study was sponsored by the Royal Brompton \& Harefield NHS Foundation Trust and funded by a grant from Innovate UK (1101_CRD_MED_IBD_61188) with contribution in kind from GlaxoSmithKline (GSK), a consortium partner. The follow-up study was funded by Novartis and the entire project was supported by the NIHR Respiratory Biomedical Research Unit at the Royal Brompton \& Harefield NHS Foundation Trust and Imperial College, which part-fund Michael I. Polkey's salary. Funding information for this article has been deposited with the Crossref Funder Registry.

Conflict of interest: Disclosures can be found alongside this article at erj.ersjournals.com

\section{References}

1 Swallow EB, Reyes D, Hopkinson NS, et al. Quadriceps strength predicts mortality in patients with moderate to severe chronic obstructive pulmonary disease. Thorax 2007; 62: 115-120.

2 Vanfleteren LE, Spruit MA, Groenen M, et al. Clusters of comorbidities based on validated objective measurements and systemic inflammation in patients with chronic obstructive pulmonary disease. Am J Respir Crit Care Med 2013; 187: 728-735.

3 Mohan D, Gale NS, McEniery CM, et al. Evaluating the role of inflammation in chronic airways disease: the ERICA study. COPD 2014; 11: 552-559.

4 Mohan D, Lewis A, Patel MS, et al. Using laser capture microdissection to study fiber specific signaling in locomotor muscle in COPD: a pilot study. Muscle Nerve 2017; 55: 902-912.

5 Hopkinson NS, Tennant RC, Dayer MJ, et al. A prospective study of decline in fat free mass and skeletal muscle strength in chronic obstructive pulmonary disease. Respir Res 2007; 8: 25.

6 Bernabeu-Mora R, Medina-Mirapeix F, Llamazares-Herran E, et al. The short physical performance battery is a discriminative tool for identifying patients with COPD at risk of disability. Int J Chron Obstruct Pulmon Dis 2015; 10: $2619-2626$.

7 Martinez-Gomez D, Bandinelli S, Del-Panta V, et al. Three-year changes in physical activity and decline in physical performance over 9 years of follow-up in older adults: the Invecchiare in Chianti Study. J Am Geriatr Soc 2017; 65: 1176-1182.

8 Patel MS, Mohan D, Andersson YM, et al. Phenotypic characteristics associated with reduced short physical performance battery score in COPD. Chest 2014; 145: 1016-1024.

9 Jones SE, Kon SS, Canavan JL, et al. The five-repetition sit-to-stand test as a functional outcome measure in COPD. Thorax 2013; 68: 1015-1020.

10 Canavan JL, Maddocks M, Nolan CM, et al. Functionally relevant cut point for isometric quadriceps muscle strength in chronic respiratory disease. Am J Respir Crit Care Med 2015; 192: 395-397. 